Bull. Austral. Math. Soc.

Vol. 41 (1990) [307-311]

\title{
THE OTHER LAW OF THE ITERATED LOGARITHM FOR MARTINGALES
}

\section{R. M. Huggins}

A Skorokhod embedding approach is used to give the other law of the iterated logarithm for square integrable martingales.

\section{INTRODUCTION}

Let $\left\{S_{n}, \mathcal{F}_{n}: n \geqslant 1\right\}$ be a square integrable martingale, that is $E S_{n}^{2}<\infty$ for all $n$, on a probability space $(\Omega, \mathcal{F}, P)$ and define $V_{n}^{2}=\sum_{j=1}^{n} E\left(X_{j}^{2} \mid \mathcal{F}_{j-1}\right)$ where $X_{n}=S_{n}-S_{n-1}$. Let $M_{n}=\max _{1 \leqslant k \leqslant n}\left|S_{k}\right|$. We are concerned here with conditions under which the other law of the iterated logarithm, that is,

$$
\liminf _{n \rightarrow \infty} \frac{M_{n}}{\left(V_{n}^{2} / \log _{2} V_{n}^{2}\right)^{\frac{1}{2}}}=\frac{\pi}{8^{\frac{1}{2}}} \text { a.s. }
$$

holds. Whilst some such conditions may be found using Theorem 6.1 of Jain, Jogdeo and Stout [2] these results depend on a sharper version of Strassen's almost sure invariance principle. A comparison with the result of Jain and Pruitt [3] when $S_{n}$ is the sum of independent and identically distributed random variables motivates us to show that (1) holds under at most the conditions of the ordinary functional law of the iterated logarithm of, for example, Hall and Heyde [1]. Here we use a Skorokhod embedding approach to extend the methods of Jain and Pruitt [3] to the martingale case. We commence with a study of the time changed Brownian motion.

\section{THE OTHER LAW OF THE ITERATED LOGARITHM FOR TIME CHANGED BROWNIAN MOTION}

For a sequence $\left\{T_{n}: n \geqslant 1\right\}$ of positive random variables and a standard Brownian motion $\{B(t): t \geqslant 0\}$, all defined on the same probability space, let $S_{n}=B\left(T_{n}\right)$ and

$$
M_{n}=\max _{1 \leqslant k \leqslant n}\left|S_{k}\right|=\max _{1 \leqslant k \leqslant n}\left|B\left(T_{k}\right)\right|
$$

Received 3 May 1989

Copyright Clearance Centre, Inc. Serial-fee code: 0004-9729/90 \$A2.00+0.00. 
THEOREM 1. If

$$
T_{n} \uparrow \infty \text { a.s. }, T_{n} / T_{n+1} \rightarrow 1 \text { a.s. }
$$

then

$$
\liminf _{n \rightarrow \infty} \frac{M_{n}}{\left(T_{n} / \log _{2} T_{n}\right)^{\frac{1}{2}}}=\frac{\pi}{8^{\frac{1}{2}}} \text { a.s. }
$$

Proof: We proceed via several lemmas.

Lemma 1. Under the conditions of the Theorem,

$$
\liminf _{n \rightarrow \infty} \frac{M_{n}}{\left(T_{n} / \log _{2} T_{n}\right)^{\frac{1}{2}}} \leqslant \frac{\pi}{8^{\frac{1}{2}}} \text { a.s. }
$$

Proof: Let $r_{n}=\max \left\{j: T_{j} \leqslant n^{n}\right\}$. From Lemma 1 of Jain and Pruitt [3] we have

so that

$$
\liminf _{n \rightarrow \infty}\left(n^{n} / \log _{2} n^{n}\right)^{-\frac{1}{2}} \max _{0 \leqslant s \leqslant n^{n}}|B(s)| \leqslant \frac{\pi}{8^{\frac{1}{2}}} \text { a.s. }
$$

and hence

$$
\liminf _{n \rightarrow \infty}\left(n^{n} / \log _{2} n^{n}\right)^{-\frac{1}{2}} \max _{0 \leqslant s \leqslant T_{r_{n}}}|B(s)| \leqslant \frac{\pi}{8^{\frac{1}{2}}} \text { a.s. }
$$

$$
\liminf _{n \rightarrow \infty}\left(n^{n} / \log _{2} n^{n}\right)^{-\frac{1}{2}} M_{\tau_{n}} \leqslant \frac{\pi}{8^{\frac{1}{3}}} \text { a.s. }
$$

which yields the lemma once it is observed that $n^{-n} T_{r_{n}} \rightarrow 1$ a.s.

Now define for $\alpha>1$,

$$
p_{n}=\max \left\{j: T_{j} \leqslant \alpha^{n}\right\}
$$

LEMma 2. Under the condition of the Theorem, for $c<\left(\pi / 8^{1 / 2}\right)$,

$$
P\left(M_{p_{n}}<c\left[\frac{\alpha^{n}}{\log _{2} \alpha^{n}}\right]^{\frac{1}{2}} \text { i.o }\right)=0 .
$$

Proof: As in Jain and Pruitt [3], choose

$$
\xi \in\left(c, \pi / 8^{1 / 2}\right), \eta \in\left(1, \pi^{2} / 8 \xi^{2}\right)
$$

and then take $\beta$ so large that

$$
2^{1 / \beta}<e^{-\eta+\pi^{2} / 8 \xi^{2}}
$$

Let $\nu=\left[\beta^{n} / \log _{2} \alpha^{n}\right]$ and $N=\left[\log _{1} \alpha^{n} / \beta\right]$. 
Now

$$
\begin{aligned}
& \left\{M_{p_{n}}<c\left(\frac{\alpha^{n}}{\log _{2} \alpha^{n}}\right)^{\frac{1}{2}}\right\} \\
& =\left\{\max _{1 \leqslant k \leqslant p_{n}}\left|B\left(T_{k}\right)\right|<c\left(\frac{\alpha^{n}}{\log _{2} \alpha^{n}}\right)^{\frac{1}{2}}\right\} \\
& C\left\{\sup _{0 \leqslant 0 \leqslant T_{p_{n}}}|B(s)|<c\left(\frac{\alpha^{n}}{\log _{2} \alpha^{n}}\right)^{\frac{1}{2}}\right\} \\
& C\left\{\sup _{0 \leqslant s \leqslant \alpha^{n}}|B(s)|<c\left(\frac{\alpha^{n}}{\log _{2} \alpha^{n}}\right)^{\frac{1}{2}}\right\} \\
& \subset \bigcap_{k=1}^{N}\left\{\sup _{(k-1) \nu<s \leqslant k \nu}|B(s)|<c\left(\frac{\alpha^{n}}{\log _{2} \alpha^{n}}\right)^{\frac{1}{2}}\right\} \\
& \subset \bigcap_{k=1}^{N}\left\{B(s)-B((k-1) \nu) \in\left(-\frac{\xi}{\beta^{\frac{1}{2}}} \nu^{\frac{1}{2}}, \frac{\xi}{\beta^{\frac{1}{2}}} \nu^{\frac{1}{2}}\right)-B((k-1) \nu),\right. \\
& \text { for all }(k-1) \nu<s \leqslant k \nu\}
\end{aligned}
$$

so that using the Markov property and well-known estimates, following Jain and Pruitt $[3]$,

$$
\begin{aligned}
& P\left(M_{p_{n}}<c\left(\frac{\alpha^{N}}{\log _{2} \alpha^{n}}\right)^{\frac{1}{2}}\right) \\
& \leqslant\left(2 e^{-\pi^{2} \beta / 8 \xi^{2}}\right)^{N} \\
& <K n^{-\eta}, \text { for some constant } K .
\end{aligned}
$$

The Borel-Cantelli lemma now completes the proof of Lemma 2.

To prove the Theorem observe that from Lemma 2 we have, with probability one,

$$
M_{p_{n}} \geqslant c\left(\frac{\alpha^{n}}{\log _{2} \alpha^{n}}\right)^{\frac{1}{2}}
$$

for all $n$ sufficiently large. Then if $p_{n}<k \leqslant p_{n+1}, M_{k} \geqslant M_{p_{n}} \geqslant c\left(\left(\alpha^{n}\right) /\left(\log _{2} \alpha^{n}\right)\right)^{1 / 2}$ $\geqslant c / \alpha\left(\left(T_{k}\right) /\left(\log _{2} T_{k}\right)\right)^{1 / 2}$ as $T_{p_{n}} \leqslant \alpha^{n} \leqslant T_{k} \leqslant \alpha^{n+1}$. As $c / \alpha$ may be made arbitrarily close to $\pi / 8^{1 / 2}$, this and Lemma 1 prove the Theorem. 


\section{THE OTHER LAW OF THE ITERATED LOGARITHM FOR MARTINGALES}

We now return to the martingale case discussed in the introduction.

THEOREM 2. If $V_{n}^{2} \rightarrow \infty$ a.s.,

$$
V_{n+1}^{\prime} / V_{n}^{2} \rightarrow 1 \text { a.s. }
$$

and for some $\delta>0$,

$$
\sum_{n=1}^{\infty} V_{n}^{-2(1+\delta)} E\left(\left|X_{n}\right|^{2(1+\delta)} \mid \mathcal{F}_{n-1}\right)<\infty
$$

then

$$
\liminf _{n \rightarrow \infty} \frac{M_{n}}{\left(V_{n}^{2} / \log _{2} V_{n}^{2}\right)^{\frac{1}{2}}}=\frac{\pi}{8^{\frac{1}{2}}} \text { a.s. }
$$

Proof: Using the Skorokhod embedding of, for example, Scott and Huggins [4], there exists a sequence of stopping times $\left\{T_{n} ; n \geqslant 1\right\}$ and a standard Brownian motion $\{B(t) ; t \geqslant 0\}$ such that

$$
B\left(T_{n}\right)=S_{n} \text { a.s., }
$$

and, where $t_{n}=T_{n}-T_{n-1}$, for some increasing family of $\sigma$-fields $\mathcal{G}_{n}$ with $T_{n} \in \mathcal{G}_{n}$, $\mathcal{G}_{n} \supseteq \mathcal{F}_{n}$

$$
E\left(t_{n} \mid \mathcal{G}_{n-1}\right)=E\left(X_{n}^{2} \mid \mathcal{F}_{n-1}\right) \text { a.s. }
$$

Further, for $1<p<\infty$, there exists a constant $C_{p}$ depending only on $p$ such that

$$
E\left(t_{n}^{p / 2} \mid \mathcal{G}_{n-1}\right) \leqslant C_{p} E\left(\left|X_{n}\right|^{p} \mathcal{F}_{n-1}\right) \text { a.s. }
$$

Thus Theorem 2 will follow from Theorem 1 once we show that

$$
V_{n}^{-2} T_{n} \rightarrow 1 \text { a.s. }
$$

To see that (6) does hold let

$$
Y_{n}=V_{n}^{-2}\left(t_{n}-E\left(t_{n} \mid \mathcal{G}_{n-1}\right)\right)
$$

so that $\left\{Y_{n} ; n \geqslant 1\right\}$ forms a martingale difference sequence. Then

$$
\begin{aligned}
& \sum_{n=1}^{\infty} V_{n}^{-2(1+\delta)} E\left(\left|t_{n}-E\left(t_{n} \mid \mathcal{G}_{n-1}\right)\right|^{(1+\delta)} \mid \mathcal{G}_{n-1}\right) \\
& \leqslant 2^{(2+\delta)} \sum_{n=1}^{\infty} V_{n}^{-2(1+\delta)} E\left(\left|t_{n}\right|^{1+\delta} \mid \mathcal{G}_{n-1}\right) \\
& \leqslant w^{(2+\delta)} C_{p} \sum_{n=1}^{\infty} V_{n}^{-2(1+\delta)} E\left(\left|X_{n}\right|^{2(1+\delta)} \mid \mathcal{F}_{n-1}\right) \text { a.s. } \\
& <\infty \text { a.s., using (5). }
\end{aligned}
$$

Thus by Corollary 2.8 .5 of Stout $[5] \sum_{n=1}^{\infty} V_{n}^{-2}\left(t_{n}-E\left(t_{n} \mid \mathcal{G}_{n-1}\right)\right)$ converges and the Krönecker lemma yields (6). 
Remarks. 1. Whilst the application of Theorem 2 to the case when the $X_{n}$ are independently and identically distributed random variables does not give the Theorem of Jain and Pruitt [3], their result can of course be obtained directly from Theorem 1 using the law of large numbers to verify (2).

2. As the conditions of our Theorem 1 are contained in the conditions of Theorem $A$ of Hall and Heyde [1] our results will hold under the various conditions for the law of the iterated logarithm for martingales given in their paper.

\section{REFERENCES}

[1] P.G. Hall and C.C. Heyde, 'On a unified approach to the law of the iterated logarithm for martingales', Bull Austral. Math. Soc. 14 (1976), 435-447.

[2] N.C. Jain, K. Jogdeo and W.F. Stout, 'Upper and lower functions for martingales and mixing processes', Ann. Prob. 3 (1975), 119-145.

[3] N.C. Jain and W.E. Pruitt, 'The other law of the iterated logarithm', Ann. Probab 3 (1975), 1046-1049.

[4] D.J. Scott and R.M. Huggins, 'On the embedding of processes in Brownian motion and the law of the iterated logarithm for reverse martingales.', Bull. Austral. Math. Soc. 27 (1983), 443-459.

[5] W.F. Stout, Almost Sure Convergence (Academic Press, New York, London, 1974).

Department of Statistics

La Trobe University

Bundoora, Vic. 3083

Australia 
